\title{
How Can Alternative Exercise Traditions Help Against the Background of the COVID-19 in Cancer Care? An Overview of Systematic Reviews
}

Yang Zhang $\mathbb{D}^{\prime}$

Fang Yao'

Xiaohong Kuang'

Lijuan $\mathrm{Li}^{{ }^{1}}$

Lihua Huang'

Qi Zhou'

Jiazhu Peng'

Qingyu Chang ${ }^{2}$

'Department of Nursing, Zhangjiagang TCM Hospital Affiliated to Nanjing University of Chinese Medicine, Suzhou 215600, People's Republic of China; ${ }^{2}$ Department of Surgery, Zhangjiagang TCM Hospital Affiliated to Nanjing University of Chinese Medicine, Suzhou 215600, People's Republic of China

Correspondence: Xiaohong Kuang Email zjgszyyykxh@aliyun.com
This article was published in the following Dove Press journal:

Cancer Management and Research

Purpose: To evaluate the quality of systematic reviews/meta-analyses (SR/MAs) on alternative exercise traditions in cancer care.

Methods: PubMed, Cochrane Library, Web of Science, Ovid, EBSCOhost, WanFang Database, China National Knowledge Infrastructure, and VIP Database were searched from their inception to June 30, 2020. The search used a combination of subject words and free words. The search terms included "Tai Chi," "Qigong," "Baduanjin," "Yoga," "Pilates," "Cancer," "Meta-analysis," and "Systematic review." Two researchers independently performed literature screening and data extraction and used AMSTAR scale and PRISMA statement to evaluate the methodology and the quality of the evidence.

Results: A total of 26 SR/MAs were included. The AMSTAR scale score was 5-10 points, with an average of $7.46 \pm 1.33$ points. Overall, the methodological quality was considered to be moderate. The PRISMA list score was 13-24 points, with an average of $19.19 \pm 2.86$ points. Among the papers evaluated, eight reports were relatively complete. A total of 15 papers indicated certain defects. A total of three papers showed relatively serious information defects. Conclusion: Relative to the control group, alternative exercise traditions may be effective in improving the quality of life, anxiety, depression, distress, and fatigue of cancer patients. However, they may not necessarily improve patients' sleep outcome, body mass index, and pain. During convalescence, cancer patients can be encouraged to start engaging in physical exercise, and professionals can develop appropriate exercise alternatives to ensure the expected effect of exercise while ensuring the safety of patients. The methodological quality of the systematic evaluations of the intervention effects of alternative exercise traditions on cancer patients is not satisfactory. Hence, focus should be directed to the improvement of the preliminary design scheme, publication status, literature retrieval, conflict of interest, and other aspects.

Keywords: alternative exercise traditions, cancer care, COVID-19, overview

\section{Introduction}

Cancer is one of the deadliest diseases in the 21 st century. ${ }^{1}$ It is characterized by high morbidity, high mortality, high treatment costs, and great difficulty in curing. ${ }^{2}$ It has become a global public health burden. ${ }^{3}$ In the United States alone, nearly $1,685,210$ new cancer cases were detected in 2016, and nearly 595,690 people died of cancer. ${ }^{4}$ Although treatment options have progressed well, the incidence and mortality of cancer are still increasing. ${ }^{5}$ Estimates indicate that by 2025 , the number 
of cancer patients could reach 20 million. ${ }^{4}$ Those who survive cancer may experience persistent difficulties, including treatment side effects and physical, cognitive, and sociopsychological struggles. ${ }^{6}$

The World Health Organization recently declared coronavirus disease 2019 (COVID-19) as a global health emergency. ${ }^{7}$ As malignant tumors and anticancer treatments (such as chemotherapy and surgery) reduce the immunity of cancer patients, cancer patients are more susceptible to infection than those who do not have cancer, and their prognosis is worse. ${ }^{8}$ The pandemic has changed the routine cancer care, and clinicians must adapt and find the best way to deliver patient care. ${ }^{9}$ In China, alternative exercise traditions play an important role in the fight against COVID-19. At the Wuhan Fangcang Hospital, patients with COVID-19 practiced alternative exercise traditions, such as Qigong and Tai Chi, under the guidance of Chinese medicine physicians and nurses. ${ }^{10}$ This approach not only enhances the body's ability to resist pathogens but also helps improve the mood of medical staff and patients and establish confidence in jointly defeating the epidemic. $^{11}$

In recent years, the number of related systematic reviews/meta-analyses (SR/MAs) of alternative exercise traditions for cancer patients has gradually increased. However, different SR/MAs have varying outcome indicators, literature quality, analysis methods, and levels of evidence. In general, the evaluation method is to conduct a comprehensive and systematic evaluation of the effects of alternative exercise traditions on cancer patients so as to provide a synthesis of clinical evidence and a basis for decision-making. As alternative forms of exercise are relatively easy to perform, patients with chronic diseases, such as cancer, may benefit from them, particularly in the context of the ongoing COVID-19 pandemic. This article attempts to explore this topic comprehensively to provide rehabilitation guidance and recommendations for patients in the recovery period.

\section{Materials and Methods Inclusion and Exclusion Criteria Research Design}

This research is about published SR/MAs of alternative exercise traditions for cancer.

\section{Research Objects}

Patients diagnosed with cancer are not limited by gender, age, race, time of onset, and source of cases.

\section{Intervention Measures}

The intervention measures mainly involve at least one of the following alternative exercise traditions: Tai Chi, Qigong, Baduanjin, yoga, and Pilates, all of which can be supplemented by other forms of therapy.

\section{Outcome Indicators}

The main outcome indicators include at least one of the following: 1) quality of life (QOL), 2) pain, 3) anxiety, 4) body mass index (BMI), 5) sleep outcome, 6) depression, 7) distress, and 8) fatigue.

\section{Exclusion Criteria}

The exclusion criteria are as follows: 1) duplicate publications, 2) systematic review plan, 3) systematic review without a quantitative analysis in the included original research, and 4) non-Chinese or English literature.

\section{Search Strategy}

A computer search of PubMed, Cochrane Library, Web of Science, Ovid, EBSCOhost, WanFang Database, China National Knowledge Infrastructure, and VIP Database was performed to filter and extract the SR/MAs that meet the inclusion criteria. The retrieval date was from the establishment date of each database to June 30, 2020. The search used a combination of subject words and free words. The search terms included "Tai Chi," "Qigong," "Baduanjin," "Yoga," "Pilates," "Cancer," "Meta-analysis, " and "Systematic review." Take PubMed as an example. Figure 1 shows the specific retrieval strategy.

\section{Literature Screening and Data Extraction}

Two researchers (Yang Zhang and Fang Yao) conducted two independent rounds of screening by reading the title, abstract, and full text. They then extracted the data according to a predesigned Excel data extraction table. In case of a disagreement, the researchers conducted discussions and

\#1 Tai Ji OR T'ai Chi OR Tai Chi Chuan OR Tai Chi OR Tai Chi Quan OR Tai-ji OR Taiji OR Taijiquan

\#2 Neoplasms OR Benign Neoplasms OR Cancer OR Malignancy OR Malignant Tumors OR Neoplasms OR Neoplasia OR Neoplasm OR Neoplasms OR Benign Tumors

\#3 meta-analysis OR systematic review

\#4 \#1 AND \#2 AND \#3

Figure I PubMed search strategy. 
consulted a third party to resolve the issue. The data extraction content included 1) basic information, including the first author's name, publication year, first author's country of origin, number of included articles, sample size, quality evaluation tools, intervention measures, control measures, outcome indicators, and research results; 2) methodology of SR/MAs, including the literature search, included studies, research samples, and methodological quality evaluation of included studies; and 3) statistical analysis results, mainly the quantitative analysis results for each outcome indicator.

\section{Evaluation of Methodological Quality and Evidence Quality of Included Studies}

The AMSTAR scale was used to carry out the evaluation of the methodological quality of the included SR/MAs. The PRISMA scale was utilized to evaluate the report quality. The items in the AMSTAR scale indicated "full report," "partial report," or "not reported." Fully meeting the AMSTAR item explanation and the comprehensive consideration of the unsatisfied parts will not cause significant deviations in the results; hence, the use of the label "full report," which is equivalent to 1 point. A "partial report" means that a part of the AMSTAR item meets the AMSTAR scale but suffers from major defects; the score in this case is 0.5 . The label "not reported" is counted as 0 point. The scale has a total of 11 points. The AMSTAR scale scores of 0-3 indicate low quality, scores of 4-7 indicate moderate quality, and scores of 8-11 indicate high quality. ${ }^{12,13}$

Each item of the PRISMA scale is described with 1 point for "complete report," 0.5 point for "partial report," and 0 point for "unreported"; the total score is 27 points. When the literature score is $21-27$, the report is considered to be relatively complete. When the score is $15-21$, the report is considered to be flawed. When the score is $\leq 15$, the report lacks sufficient information. ${ }^{14}$

\section{Results}

\section{Identification and Selection of Reviews}

The total number of related articles in the database was 663; 317 of these articles were retained after removing the duplicates. After reading the titles and abstracts of the remaining articles to exclude irrelevant ones, 69 documents were obtained. Reading the full text content yielded 26 articles, which were finally included in the study. The literature screening process and results are shown in Figure 2.

\section{Characteristics of Included Reviews}

The 26 SR/MAs included 22 English articles ${ }^{15-36}$ and 4 Chinese articles. ${ }^{37-40}$ The publication years ranged from 2007 to 2020, with 12 articles published in the last 5 years. Of the $26 \mathrm{SR} / \mathrm{MAs}, 13$ focused on yoga,

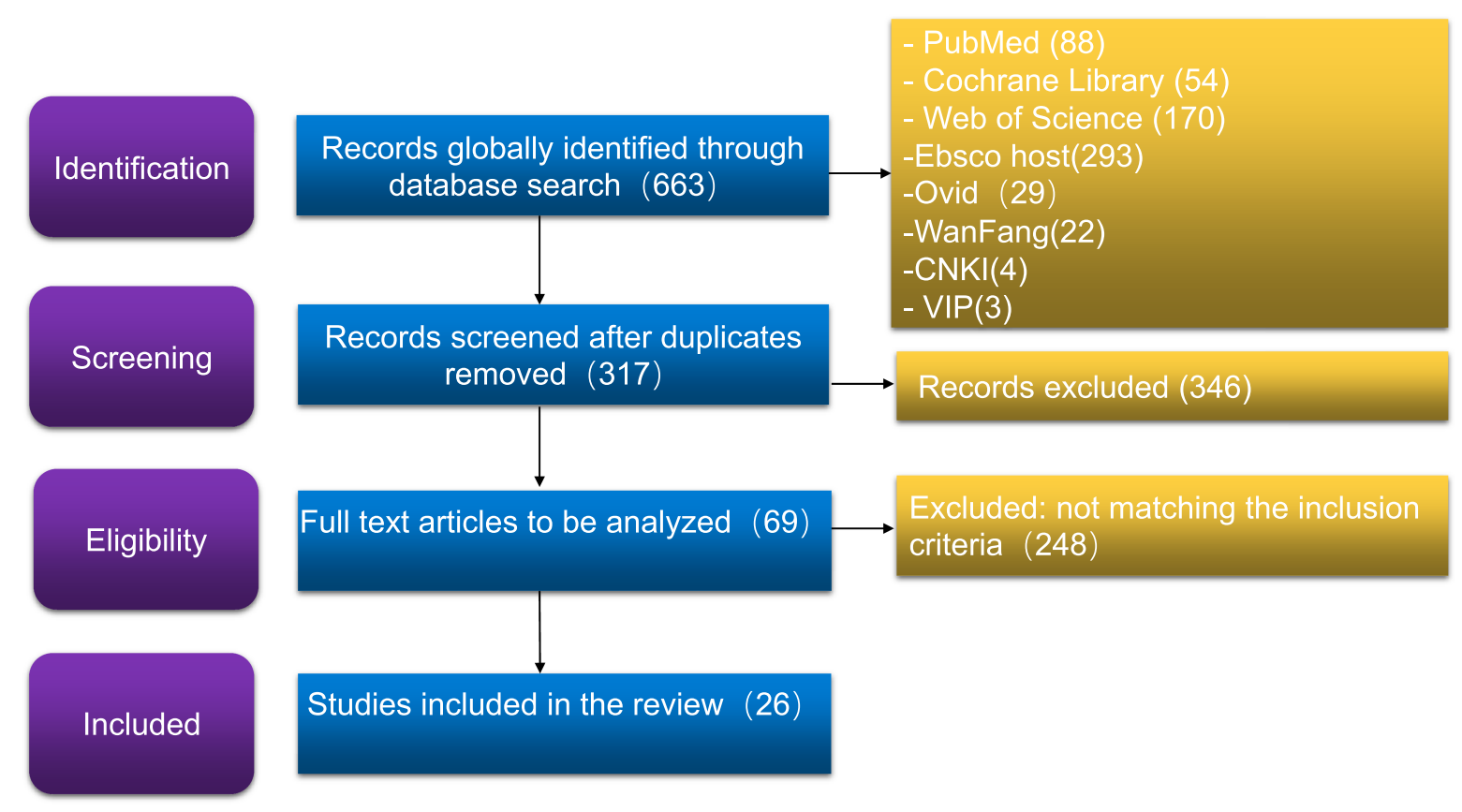

Figure 2 The PRISMA flow diagram of study inclusion in the review.

Notes: PRISMA figure adapted from Liberati A, Altman D, TetzlaffJ, et al. The PRISMA statement for reporting systematic reviews and meta-analyses of studies that evaluate health care interventions: explanation and elaboration. Journal of clinical epidemiology. 2009;62(10). Creative Commons. ${ }^{4 I}$ 
12 focused on Tai Chi, 5 focused on Qigong, and 2 focused on Pilates. All SR/MAs evaluated the methodological quality of the included original studies; 17 used the Cochrane criteria, 3 did not mention the quality criteria of their evaluation, 2 used the Jadad scale, 3 used the PEDro criteria, and 1 used the NIH Quality Assessment. The basic characteristics included in the SR/MAs are shown in Table 1. The clear outcome results of the included SR/ MAs are shown in Table 2.

\section{AMSTAR Evaluation Results}

The AMSTAR scale was used to evaluate the methodological quality of the included SR/MAs. As shown in Table 3, the AMSTAR scale scores of the $26 \mathrm{SR} / \mathrm{MAs}$ ranged from 5 points to 10 points, with an average of $7.46 \pm 1.33$ points. Among them, 16 received $\geq 8$ points, which indicated highquality studies that accounted for $61.5 \%$ of the total number; 10 of the articles were moderate-quality studies (4-7 points), and they accounted for $38.5 \%$. Generally, the methodological quality was regarded as moderate. No literature fully met the requirements of the 11 items in the AMSTAR scale. Relatively complete reports $(\geq 70 \%)$ included entry $2(80.8 \%)$, entry $5(88.5 \%)$, entry $6(100 \%)$, entry $7(96.2 \%)$, entry $8(100 \%)$, and entry $9(100 \%)$.

\section{PRISMA Evaluation Results}

As shown in Table 4, the PRISMA scores of the 26 SR/MAs ranged from 13.0 to 24.0 , with an average of $19.19 \pm 2.86$ points. Among them, 8 reports were relatively complete (21-27 points, including 27 points), accounting for $30.8 \%$; 15 reports were flawed (15-21 points, including 21 points), accounting for $57.7 \%$; 3 reports had relatively serious information deficiency ( $\leq 15$ points), accounting for $11.5 \%$. No literature fully met the requirements of the 27 items in the PRISMA scale. Report information was lacking $(<50 \%)$ in the following 13 entries: entry $2(7.7 \%)$, entry $3(30.8 \%)$, entry $5(11.5 \%)$, entry $7(34.6 \%)$, entry $8(26.9 \%)$, entry $9(30.8 \%)$, entry 10 (38.5\%), entry $12(19.2 \%)$, entry $15(38.5 \%)$, entry $16(15.4 \%)$, entry $19(30.8 \%)$, entry $22(23.1 \%)$, and entry $23(11.5 \%)$.

\section{Discussion}

\section{The Methodological Quality of the SR/} MAs of Alternative Exercise Traditions for Cancer Treatment Needs to be

\section{Improved}

This study included 26 SR/MAs of alternative exercise traditions for cancer treatment. All included SR/MAs were mainly published from 2007 to 2020 . Among these articles, 12 were published in the last 5 years; hence, the popularity of alternative exercise traditions for cancer treatment is on the rise. Only high-quality systematic reviews can provide relatively unbiased and scientific evidence for clinical practice and health decision-making. Therefore, systematic reviews need to strictly control the quality of the included studies and methodologies. In the current work, the AMSTAR scale was used to evaluate the methodological quality of the included SR/MAs. The results showed that 16 articles were of high quality, and they accounted for $61.5 \% ; 10$ articles were of moderate quality, and they accounted for $38.5 \%$.

A total of 23 papers (88.5\%) lacked preliminary design plans; 4 papers $(15.4 \%)$ did not pay attention to the repeatability of document extraction; 15 papers $(57.7 \%)$ did not search the necessary databases, such as the Cochrane Library, Web of Science, and PubMed; 22 papers $(84.6 \%)$ did not consider the unpublished status in the inclusion criteria (if gray literature was not fully considered); 3 papers (11.5\%) lacked a specific list of included and excluded studies (if specific reasons for exclusion were not included); 1 paper (3.8\%) did not evaluate and report the scientific nature of the included research; 8 papers $(30.8 \%)$ did not evaluate the publication deviation (such as a one-sided description); 14 papers $(53.8 \%)$ did not state the relevant funding sources and the presence of relevant conflicts of interest. The above methodological quality defects may affect the rigor of the system evaluation, the accuracy of the results, and the overall objectivity.

Therefore, the quality of methodology needs to be further improved. For example, if only electronic retrieval is performed and paper journals and books are not manually retrieved, then relevant articles may be missed. In such a case, the included articles may not be comprehensive enough. This deficiency may affect the accuracy of the results and reduce the quality of the methodology.

\section{The Quality of the Reports on Alternative Exercise Traditions for Cancer Treatment Needs to be Strengthened}

This study used the PRISMA scale to evaluate the quality of the reports. The results showed that 8 reports $(30.8 \%)$ were relatively complete, 15 reports $(57.7 \%)$ 
Table I Characteristics of Included SR/MAs

\begin{tabular}{|c|c|c|c|c|c|c|c|c|}
\hline $\begin{array}{l}\text { Authors, } \\
\text { Year }\end{array}$ & Country & $\begin{array}{l}\text { Number } \\
\text { of } \\
\text { Studies/ } \\
\text { Sample } \\
\text { Size }\end{array}$ & $\begin{array}{l}\text { Quality } \\
\text { Criteria }\end{array}$ & $\begin{array}{l}\text { Duration } \\
\text { of Studies }\end{array}$ & Intervention Group & Control Group & $\begin{array}{l}\text { Main } \\
\text { Outcome }\end{array}$ & $\begin{array}{l}\text { Outcome } \\
\text { Results }\end{array}$ \\
\hline $\begin{array}{l}\text { Luo et al, } \\
2020^{15}\end{array}$ & China & $15 / 885$ & $\begin{array}{l}\text { Cochrane } \\
\text { criteria }\end{array}$ & $2006-2019$ & $\begin{array}{l}\text { RRT+8-form TCC; Usual care } \\
\text { +8-form TCC; RRT + Chen- } \\
\text { style TCC; Usual care + 18- } \\
\text { form TCC; RRT + 24-form } \\
\text { simplified } \\
\text { TCC;RRT + Tai Chi Yunshou; } \\
\text { I5-move short-form of Yang- } \\
\text { style TCC }\end{array}$ & $\begin{array}{l}\text { Usual care; } \\
\text { Cognitive } \\
\text { behavioral } \\
\text { therapy; RRT; } \\
\text { Standard support } \\
\text { Therapy; } \\
\text { Psychosocial } \\
\text { support therapy }\end{array}$ & (1)(2)(3) & $\begin{array}{l}\text { TCC is effective } \\
\text { on quality of life, } \\
\text { pain and anxiety } \\
\text { in breast cancer } \\
\text { patients. }\end{array}$ \\
\hline $\begin{array}{l}\text { Lee et al, } \\
2010^{16}\end{array}$ & $\begin{array}{l}\text { South } \\
\text { Korea }\end{array}$ & $7 / 235$ & $\begin{array}{l}\text { Cochrane } \\
\text { criteria }\end{array}$ & $\begin{array}{l}2003- \\
2009\end{array}$ & $\begin{array}{l}\text { Tai Chi;Yang style;Combining } \\
\text { Yang and Sun style }\end{array}$ & $\begin{array}{l}\text { No treatment; } \\
\text { Psychosocial } \\
\text { support therapy; } \\
\text { Spiritual growth; } \\
\text { Standard health } \\
\text { Care; Walking; } \\
\text { Education } \\
\text { Program }\end{array}$ & (1) & $\begin{array}{l}\text { Tai Chi is not } \\
\text { effective on } \\
\text { quality of life for } \\
\text { supportive breast } \\
\text { cancer care. }\end{array}$ \\
\hline $\begin{array}{l}\text { Pan et al, } \\
2015^{17}\end{array}$ & China & 19/322 & $\begin{array}{l}\text { Cochrane } \\
\text { criteria }\end{array}$ & 2004-2013 & $\begin{array}{l}\text { Tai Chi; Yang-style TCC; } \\
\text { 8-form TCC }\end{array}$ & $\begin{array}{l}\text { Health education; } \\
\text { Psychosocial } \\
\text { Therapy; Standard } \\
\text { support therapy; } \\
\text { Usual care; } \\
\text { Spiritual growth } \\
\text { and standard } \\
\text { health care }\end{array}$ & (2)(4) & $\begin{array}{l}\text { Tai Chi is not } \\
\text { effective on pain } \\
\text { and BMI in breast } \\
\text { cancer patients. }\end{array}$ \\
\hline $\begin{array}{l}\text { Yan et al, } \\
2014^{18}\end{array}$ & China & $5 / 407$ & Jadad scale & 2004-2012 & $\begin{array}{l}\text { 15-move short-form of Yang- } \\
\text { style TCC; } 24 \text {-form of TCC; } \\
\text { 8-move short form of TCC }\end{array}$ & $\begin{array}{l}\text { Psychosocial } \\
\text { Therapy; Standard } \\
\text { support therapy; } \\
\text { Usual care; } \\
\text { Standard } \\
\text { rehabilitation }\end{array}$ & (1)(4) & $\begin{array}{l}\text { Tai Chi is not } \\
\text { effective on quality } \\
\text { of life (except } \\
\text { emotional well- } \\
\text { being) and BMI in } \\
\text { breast cancer } \\
\text { patients. }\end{array}$ \\
\hline $\begin{array}{l}\text { Zhang } \\
\text { et al, } \\
2012^{19}\end{array}$ & China & $6 / 382$ & $\begin{array}{l}\text { Cochrane } \\
\text { criteria }\end{array}$ & $2007-2012$ & $\begin{array}{l}\text { Hatha yoga; Restorative yoga; } \\
\text { lyengar yoga; Vini yoga; } \\
\text { Patanjali's yoga }\end{array}$ & $\begin{array}{l}\text { Wait-list; } \\
\text { Nontreatment }\end{array}$ & (1)(3)(5)(6)(7) & $\begin{array}{l}\text { Yoga is effective } \\
\text { on quality of life, } \\
\text { but not effective } \\
\text { on anxiety, } \\
\text { depression, } \\
\text { distress and sleep } \\
\text { outcome in } \\
\text { women with } \\
\text { breast cancer }\end{array}$ \\
\hline
\end{tabular}

(Continued) 
Table I (Continued).

\begin{tabular}{|c|c|c|c|c|c|c|c|c|}
\hline $\begin{array}{l}\text { Authors, } \\
\text { Year }\end{array}$ & Country & $\begin{array}{l}\text { Number } \\
\text { of } \\
\text { Studies/ } \\
\text { Sample } \\
\text { Size }\end{array}$ & $\begin{array}{l}\text { Quality } \\
\text { Criteria }\end{array}$ & $\begin{array}{l}\text { Duration } \\
\text { of Studies }\end{array}$ & Intervention Group & Control Group & $\begin{array}{l}\text { Main } \\
\text { Outcome }\end{array}$ & $\begin{array}{l}\text { Outcome } \\
\text { Results }\end{array}$ \\
\hline $\begin{array}{l}\text { Buffart } \\
\text { et al, } \\
2012^{20}\end{array}$ & Netherlands & $13 / 695$ & $\begin{array}{l}\text { Not } \\
\text { mentioned }\end{array}$ & $\begin{array}{l}2003- \\
2011\end{array}$ & $\begin{array}{l}\text { Integrated yoga program; } \\
\text { Viniyoga; Yoga asanas; } \\
\text { Savasana; Tibetan yoga }\end{array}$ & $\begin{array}{l}\text { Non-exercise; } \\
\text { Wait-list }\end{array}$ & (3)(5)(6)(7)(8) & $\begin{array}{l}\text { Yoga is effective } \\
\text { on anxiety, } \\
\text { depression and } \\
\text { distress, but not } \\
\text { effective on sleep } \\
\text { outcome and } \\
\text { fatigue in cancer } \\
\text { patients and } \\
\text { survivors. }\end{array}$ \\
\hline $\begin{array}{l}\text { Cramer } \\
\text { et al, } \\
2012^{21}\end{array}$ & Germany & $12 / 742$ & $\begin{array}{l}\text { Cochrane } \\
\text { criteria }\end{array}$ & $2003-2011$ & $\begin{array}{l}\text { lyengar Yoga; Integrated yoga; } \\
\text { Yoga of Awareness; Patanjali's } \\
\text { yoga Sutras; Integrated yoga; } \\
\text { Hatha yoga; Restorative yoga; } \\
\text { Viniyoga }\end{array}$ & $\begin{array}{l}\text { Wail-list; } \\
\text { Supportive } \\
\text { counseling and } \\
\text { advice to take } \\
\text { light exercise; } \\
\text { Health education }\end{array}$ & (1)(3) (6) & $\begin{array}{l}\text { Yoga is effective } \\
\text { on quality of life, } \\
\text { and short-term } \\
\text { yoga is effective } \\
\text { on anxiety and } \\
\text { depression in } \\
\text { breast cancer } \\
\text { patients. }\end{array}$ \\
\hline $\begin{array}{l}\text { Dong } \\
\text { et al, } \\
2019^{22}\end{array}$ & China & $12 / 2183$ & $\begin{array}{l}\text { Cochrane } \\
\text { criteria }\end{array}$ & $2009-2018$ & $\begin{array}{l}\text { Hatha yoga+home-based } \\
\text { yoga; Integrated yoga; } \\
\text { Restorative yoga; lyengar } \\
\text { yoga; Viniyoga+home-based } \\
\text { yoga; Satyananda yoga; } \\
\text { Anusara yoga; Classical yoga; } \\
\text { Home-based yoga; Classical } \\
\text { yoga; Dru yoga+home-based } \\
\text { breathing and relaxation } \\
\text { exercise; Tibetan yoga } \\
\text { +booster class +home-based } \\
\text { practice; Yoga +usual care; } \\
\text { Yoga+Aerobic exercise }\end{array}$ & $\begin{array}{l}\text { Standard care; } \\
\text { Supportive } \\
\text { counseling; Wait- } \\
\text { list } \\
\text { Non-intervention; } \\
\text { Health education; } \\
\text { Usual self-care; } \\
\text { Usual care; } \\
\text { Strengthening } \\
\text { exercise } \\
\text { Conventional } \\
\text { physical exercise; } \\
\text { Aerobic exercise }\end{array}$ & (8) & $\begin{array}{l}\text { Yoga is effective } \\
\text { on fatigue in } \\
\text { patients with } \\
\text { breast cancer. }\end{array}$ \\
\hline $\begin{array}{l}\text { Ford et al, } \\
2020^{23}\end{array}$ & USA & $17 / 666$ & $\begin{array}{l}\text { NIH } \\
\text { Quality } \\
\text { Assessment }\end{array}$ & $\begin{array}{l}2000- \\
2017\end{array}$ & Taichi; Qigong; Yoga & $\begin{array}{l}\text { Usual care; Wait- } \\
\text { list; Education } \\
\text { groups; Attention } \\
\text { controls }\end{array}$ & (1) & $\begin{array}{l}\text { TaiChi/Qigong } \\
\text { and Yoga are } \\
\text { effective on } \\
\text { quality of life in } \\
\text { male cancer } \\
\text { survivors. }\end{array}$ \\
\hline $\begin{array}{l}\text { Hashimi } \\
\text { et al, } \\
2019^{24}\end{array}$ & Canada & $8 / 545$ & $\begin{array}{l}\text { Not } \\
\text { mentioned }\end{array}$ & $2008-2018$ & $\begin{array}{l}\text { Vivekananda Yoga } \\
\text { Anusandhana Samsthana; } \\
\text { Hatha yoga; Anusandhana } \\
\text { Samsthana; } \\
\text { Tibetan yoga; lyengar yoga; } \\
\text { Integrated Yoga; Classic yoga }\end{array}$ & $\begin{array}{l}\text { Stretching; } \\
\text { Standard exercise; } \\
\text { Physical exercise; } \\
\text { Shoulder } \\
\text { exercise; Strength } \\
\text { training; } \\
\text { Physical exercise; } \\
\text { Aerobic exercise }\end{array}$ & (1) & $\begin{array}{l}\text { Yoga is not } \\
\text { effective on } \\
\text { quality of life of } \\
\text { women with } \\
\text { breast cancer. }\end{array}$ \\
\hline
\end{tabular}

(Continued) 
Table I (Continued).

\begin{tabular}{|c|c|c|c|c|c|c|c|c|}
\hline $\begin{array}{l}\text { Authors, } \\
\text { Year }\end{array}$ & Country & $\begin{array}{l}\text { Number } \\
\text { of } \\
\text { Studies/ } \\
\text { Sample } \\
\text { Size }\end{array}$ & $\begin{array}{l}\text { Quality } \\
\text { Criteria }\end{array}$ & $\begin{array}{l}\text { Duration } \\
\text { of Studies }\end{array}$ & Intervention Group & Control Group & $\begin{array}{l}\text { Main } \\
\text { Outcome }\end{array}$ & $\begin{array}{l}\text { Outcome } \\
\text { Results }\end{array}$ \\
\hline $\begin{array}{l}\text { Harder } \\
\text { et al, } \\
2012^{25}\end{array}$ & UK & $18 / 164$ & $\begin{array}{l}\text { PEDro } \\
\text { criteria }\end{array}$ & $\begin{array}{l}2006- \\
2012\end{array}$ & $\begin{array}{l}\text { Integrated yoga; Lyengar yoga; } \\
\text { Hatha yoga; Restorative yoga; } \\
\text { Yoga of Awareness; Lyengar } \\
\text { yoga; Integrated yoga; } \\
\text { Modified Hatha yoga; Patanjali } \\
\text { yoga }\end{array}$ & $\begin{array}{l}\text { Supportive } \\
\text { therapy; Wait-list; } \\
\text { Health education; } \\
\text { Standard care }\end{array}$ & (1) & $\begin{array}{l}\text { Yoga may be } \\
\text { a useful practice } \\
\text { in improving the } \\
\text { quality of life of } \\
\text { women with } \\
\text { breast cancer. }\end{array}$ \\
\hline $\begin{array}{l}\text { Lee } \\
\text { et al, } \\
2007^{26}\end{array}$ & UK & $4 / 226$ & $\begin{array}{l}\text { Jadad } \\
\text { Assessment }\end{array}$ & $2003-2006$ & Tai Chi & $\begin{array}{l}\text { Walking exercise; } \\
\text { Psychosocial } \\
\text { support:Education } \\
\text { program }\end{array}$ & (1) & $\begin{array}{l}\text { Tai Chi is not } \\
\text { effective on } \\
\text { quality of life for } \\
\text { cancer. }\end{array}$ \\
\hline $\begin{array}{l}\text { Pan } \\
\text { et al, } \\
2015^{27}\end{array}$ & China & $16 / 930$ & $\begin{array}{l}\text { Cochrane } \\
\text { criteria }\end{array}$ & $2007-2012$ & $\begin{array}{l}\text { Integrated yoga;lyengar yoga; } \\
\text { Yoga of awareness Program; } \\
\text { Patanjali’s yoga; Modified } \\
\text { yoga; } \\
\text { Restorative yoga; Viniyoga }\end{array}$ & $\begin{array}{l}\text { Supportive } \\
\text { therapy; Wait-list; } \\
\text { Health education; } \\
\text { Usual care; } \\
\text { Standard } \\
\text { physiotherapy; } \\
\text { Brief supportive } \\
\text { therapy }\end{array}$ & (1)(3)(5)(6) & $\begin{array}{l}\text { Yoga is effective } \\
\text { on sleep } \\
\text { outcome,quality } \\
\text { of life, } \\
\text { anxiety and } \\
\text { depression, but } \\
\text { not effective on } \\
\text { fatigue } \\
\text { for patients } \\
\text { recovering from } \\
\text { breast cancer. }\end{array}$ \\
\hline $\begin{array}{l}\text { Wang } \\
\text { et al, } \\
2020^{28}\end{array}$ & China & 19/1832 & $\begin{array}{l}\text { Cochrane } \\
\text { criteria }\end{array}$ & $\begin{array}{l}2009 \\
2019\end{array}$ & $\begin{array}{l}\text { lyengar Yoga; Asanas yoga; } \\
\text { Viniyoga; Restorative yoga; } \\
\text { Hatha yoga; Tibetan yoga; } \\
\text { Mindful yoga; Yoga breathing } \\
\text { exercise in warm water }\end{array}$ & $\begin{array}{l}\text { Wait-list; Health } \\
\text { education; } \\
\text { Usual care; Social } \\
\text { support }\end{array}$ & (5) & $\begin{array}{l}\text { Yoga is an } \\
\text { effective } \\
\text { supportive } \\
\text { treatment for } \\
\text { cancer in sleep } \\
\text { outcome. }\end{array}$ \\
\hline $\begin{array}{l}\text { Sadja } \\
\text { et al, } \\
2013^{29}\end{array}$ & USA & $10 / 583$ & $\begin{array}{l}\text { Not } \\
\text { mentioned }\end{array}$ & $\begin{array}{l}2004- \\
2012\end{array}$ & $\begin{array}{l}\text { lyengar yoga; Tibetan yoga; } \\
\text { Yoga of awareness; Patanjali's } \\
\text { yoga tradition; Based on } \\
\text { Hatha; Integrated yoga } \\
\text { program }\end{array}$ & $\begin{array}{l}\text { Wait-list; Health } \\
\text { education }\end{array}$ & (8) & $\begin{array}{l}\text { Yoga may be } \\
\text { beneficial for } \\
\text { reducing fatigue } \\
\text { in women with } \\
\text { breast cancer. }\end{array}$ \\
\hline $\begin{array}{l}\text { Liu } \\
\text { et al, } \\
2020^{30}\end{array}$ & China & $16 / 1268$ & $\begin{array}{l}\text { PEDro } \\
\text { criteria }\end{array}$ & $\begin{array}{l}2003- \\
2017\end{array}$ & $\begin{array}{l}\text { Tai Chi; Tai Chi+Standard } \\
\text { rehabilitation training }\end{array}$ & $\begin{array}{l}\text { Cognitive } \\
\text { behavior therapy; } \\
\text { Standard } \\
\text { rehabilitation } \\
\text { training; } \\
\text { Usual care; } \\
\text { Walking; } \\
\text { Psychosocial } \\
\text { support therapy; } \\
\text { Spiritual growth } \\
\text { group }\end{array}$ & $\begin{array}{l}\text { (1)(4)(5)(6) } \\
\text { (8) }\end{array}$ & $\begin{array}{l}\text { Tai Chi is } \\
\text { effective on } \\
\text { quality of life, but } \\
\text { not effective on } \\
\text { sleep outcome, } \\
\text { BMI, depression } \\
\text { and fatigue in } \\
\text { breast cancer } \\
\text { patients. }\end{array}$ \\
\hline
\end{tabular}

(Continued) 
Table I (Continued).

\begin{tabular}{|c|c|c|c|c|c|c|c|c|}
\hline $\begin{array}{l}\text { Authors, } \\
\text { Year }\end{array}$ & Country & $\begin{array}{l}\text { Number } \\
\text { of } \\
\text { Studies/ } \\
\text { Sample } \\
\text { Size }\end{array}$ & $\begin{array}{l}\text { Quality } \\
\text { Criteria }\end{array}$ & $\begin{array}{l}\text { Duration } \\
\text { of Studies }\end{array}$ & Intervention Group & Control Group & $\begin{array}{l}\text { Main } \\
\text { Outcome }\end{array}$ & $\begin{array}{l}\text { Outcome } \\
\text { Results }\end{array}$ \\
\hline $\begin{array}{l}\text { Espíndula } \\
\text { et al, } \\
2017^{31}\end{array}$ & Brazil & $4 / 150$ & $\begin{array}{l}\text { Cochrane } \\
\text { criteria }\end{array}$ & $2010-2016$ & Pilates; Pilates+home exercise & $\begin{array}{l}\text { Home exercise; } \\
\text { No exercise }\end{array}$ & (2)(8) & $\begin{array}{l}\text { Pilates may be } \\
\text { beneficial for } \\
\text { reducing pain and } \\
\text { fatigue of breast } \\
\text { cancer patients. }\end{array}$ \\
\hline $\begin{array}{l}\text { Carral } \\
\text { et al, } \\
2018^{32}\end{array}$ & Spain & $4 / 246$ & $\begin{array}{l}\text { Cochrane } \\
\text { criteria }\end{array}$ & $2008-2013$ & $\begin{array}{l}\text { Pilates exercise+home pilates; } \\
\text { Pilates mat exercise+ } \\
\text { information and home } \\
\text { drainage and walking exercise; } \\
\text { Pilates mat exercise; Pilates } \\
\text { MVe Fitnesess Chair; Pilates } \\
\text { mat exercise+Pilates-based } \\
\text { theraband exercises; Pilates+ } \\
\text { home standard lymphedema } \\
\text { exercises }\end{array}$ & $\begin{array}{l}\text { Information and } \\
\text { drainage and } \\
\text { walking exercise; } \\
\text { No exercise; } \\
\text { Information and } \\
\text { drainage and } \\
\text { walking exercise; } \\
\text { Traditional } \\
\text { resistance } \\
\text { training; } \\
\text { Conventional } \\
\text { exercise and } \\
\text { breast prosthesis } \\
\text { counseling; } \\
\text { Exercise } \\
\text { programme; } \\
\text { Lumbopelvic } \\
\text { stability }\end{array}$ & (1)(2) & $\begin{array}{l}\text { Pilates has } \\
\text { a certain effect } \\
\text { on quality of life } \\
\text { and pain for } \\
\text { women with } \\
\text { breast cancer. }\end{array}$ \\
\hline $\begin{array}{l}\text { Zeng } \\
\text { et al, } \\
2014^{33}\end{array}$ & China & $13 / 592$ & $\begin{array}{l}\text { Cochrane } \\
\text { criteria }\end{array}$ & $2003-2013$ & $\begin{array}{l}\text { Taichi; Guolin new qigong; } \\
\text { Yang style of taichi; Medical } \\
\text { qigong }\end{array}$ & $\begin{array}{l}\text { Health education; } \\
\text { Wait-list; } \\
\text { Walking exercise; } \\
\text { Psychosocial } \\
\text { support; Usual } \\
\text { medical care; } \\
\text { Standard support } \\
\text { therapy }\end{array}$ & (1)(3)(4)(6) & $\begin{array}{l}\text { Qigong/Tai Chi } \\
\text { are effective on } \\
\text { quality of life and } \\
\text { anxiety,but not } \\
\text { effective on BMI } \\
\text { and depression of } \\
\text { cancer patients. }\end{array}$ \\
\hline $\begin{array}{l}\text { Wayne } \\
\text { et al, } \\
2017^{34}\end{array}$ & USA & $15 / 1283$ & $\begin{array}{l}\text { Cochrane } \\
\text { criteria }\end{array}$ & $2008-2013$ & Taichi; Qigong & $\begin{array}{l}\text { Psychosocial } \\
\text { support; Usual } \\
\text { care; } \\
\text { Health education; } \\
\text { No treatment; } \\
\text { Spiritual growth; } \\
\text { Stretching } \\
\text { exercise }\end{array}$ & (1)(2)(5)(6) & $\begin{array}{l}\text { Qigong/Tai Chi } \\
\text { are effective on } \\
\text { sleep outcome, } \\
\text { quality of life, } \\
\text { depression and } \\
\text { fatigue,but not } \\
\text { effective on pain } \\
\text { of cancer } \\
\text { patients. }\end{array}$ \\
\hline
\end{tabular}

(Continued) 
Table I (Continued).

\begin{tabular}{|c|c|c|c|c|c|c|c|c|}
\hline $\begin{array}{l}\text { Authors, } \\
\text { Year }\end{array}$ & Country & $\begin{array}{l}\text { Number } \\
\text { of } \\
\text { Studies/ } \\
\text { Sample } \\
\text { Size }\end{array}$ & $\begin{array}{l}\text { Quality } \\
\text { Criteria }\end{array}$ & $\begin{array}{l}\text { Duration } \\
\text { of Studies }\end{array}$ & Intervention Group & Control Group & $\begin{array}{l}\text { Main } \\
\text { Outcome }\end{array}$ & $\begin{array}{l}\text { Outcome } \\
\text { Results }\end{array}$ \\
\hline $\begin{array}{l}\text { Zeng } \\
\text { et al, } \\
2019^{35}\end{array}$ & China & $12 / 915$ & $\begin{array}{l}\text { Cochrane } \\
\text { criteria }\end{array}$ & $2014-2018$ & $\begin{array}{l}\text { Taichi; Qigong; Taichi } \\
\text { +Qigong; } \\
\text { Medical qigong }\end{array}$ & $\begin{array}{l}\text { Usual care; } \\
\text { Support groups; } \\
\text { Wait-list }\end{array}$ & (1)(3)(5) 8 & $\begin{array}{l}\text { Qigong/Tai Chi } \\
\text { are effective on } \\
\text { sleep outcome } \\
\text { and fatigue, but } \\
\text { not effective on } \\
\text { quality of life, } \\
\text { anxiety, } \\
\text { depression of } \\
\text { cancer patients. }\end{array}$ \\
\hline $\begin{array}{l}\text { Lin et al, } \\
2011^{36}\end{array}$ & China & $10 / 788$ & $\begin{array}{l}\text { PEDro } \\
\text { criteria }\end{array}$ & 2000-2009 & $\begin{array}{l}\text { Restorative yoga; Integrated } \\
\text { yoga; Gentle yoga; Hatha } \\
\text { yoga; Asanas+shevasana; } \\
\text { Tibetan yoga }\end{array}$ & $\begin{array}{l}\text { Wait-list; Support } \\
\text { therapy }\end{array}$ & (3)(6) 7 & $\begin{array}{l}\text { Yoga is effective } \\
\text { on anxiety, } \\
\text { depression and } \\
\text { distress of cancer } \\
\text { patients. }\end{array}$ \\
\hline $\begin{array}{l}\text { Pan et al, } \\
2016^{37}\end{array}$ & China & 12/1979 & $\begin{array}{l}\text { Cochrane } \\
\text { criteria }\end{array}$ & 2004-2013 & $\begin{array}{l}\text { 19-move short-form of TCC; } \\
\text { Qigong + Short-form of Yang- } \\
\text { style TCC; Short-form of } \\
\text { Yang-style TCC; } 24 \text {-form of } \\
\text { TCC }\end{array}$ & $\begin{array}{l}\text { Rehabilitation } \\
\text { nursing; Health } \\
\text { education; } \\
\text { Psychosocial } \\
\text { therapy; } \\
\text { Standard } \\
\text { rehabilitation; } \\
\text { Usual care; Music } \\
\text { Rehabilitation } \\
\text { exercise }\end{array}$ & (1)(2)(4) & $\begin{array}{l}\text { Tai Chi is } \\
\text { effective on BMI, } \\
\text { but not effective } \\
\text { on quality of life } \\
\text { and pain of } \\
\text { postoperative } \\
\text { patients } \\
\text { with breast } \\
\text { cancer. }\end{array}$ \\
\hline $\begin{array}{l}\text { Yan et al, } \\
2013^{38}\end{array}$ & China & $4 / 169$ & $\begin{array}{l}\text { Cochrane } \\
\text { criteria }\end{array}$ & $2003-2010$ & $\begin{array}{l}\text { Yang-style TCC; Short form } \\
\text { of TCC; } 24 \text {-move short form } \\
\text { of TCC }\end{array}$ & $\begin{array}{l}\text { Psychosocial } \\
\text { Therapy; Walking; } \\
\text { Standard Therapy; } \\
\text { Usual } \\
\text { rehabilitation }\end{array}$ & (1)(4) & $\begin{array}{l}\text { Tai Chi is not } \\
\text { effective on } \\
\text { quality of life and } \\
\text { BMl in breast } \\
\text { cancer patients. }\end{array}$ \\
\hline $\begin{array}{l}\text { Zhang } \\
\text { et al, } \\
2015^{39}\end{array}$ & China & $9 / 623$ & $\begin{array}{l}\text { Cochrane } \\
\text { criteria }\end{array}$ & $2007-2014$ & $\begin{array}{l}\text { Anusara yoga; Lyengar yoga; } \\
\text { Patanjali's yoga; Restorative } \\
\text { yoga; Hatha yoga; Viniyoga }\end{array}$ & $\begin{array}{l}\text { Usual care; Health } \\
\text { education; One- } \\
\text { to-one meeting }\end{array}$ & (8) & $\begin{array}{l}\text { Yoga is effective } \\
\text { on fatigue of } \\
\text { breast cancer } \\
\text { patients. }\end{array}$ \\
\hline $\begin{array}{l}\text { Wu } \\
\text { et al, } \\
2018^{40}\end{array}$ & China & $7 / 671$ & $\begin{array}{l}\text { Cochrane } \\
\text { criteria }\end{array}$ & 2015-2017 & Yoga & $\begin{array}{l}\text { Wait-list; Health } \\
\text { education; Usual } \\
\text { care; Social } \\
\text { support }\end{array}$ & (1)(3)(5)(6) & $\begin{array}{l}\text { Yoga is effective } \\
\text { on quality of life, } \\
\text { anxiety and } \\
\text { depression, but } \\
\text { not effective on } \\
\text { sleep outcome in } \\
\text { patients with } \\
\text { breast cancer. }\end{array}$ \\
\hline
\end{tabular}

Notes: TCC, Tai Chi; (1)quality of life;(2)pain;(3)anxiety;(4)BMl;(5)sleep outcome; (6) depression; (7) distress; (8) fatigue. 
Table 2 Outcome Results of Included SR/MAs

\begin{tabular}{|c|c|c|c|c|c|c|c|c|}
\hline Outcome & Authors, Year & $\begin{array}{l}\text { Outcome } \\
\text { Results }\end{array}$ & Outcome & Authors, Year & $\begin{array}{l}\text { Outcome } \\
\text { Results }\end{array}$ & Outcome & Authors, Year & $\begin{array}{l}\text { Outcome } \\
\text { Results }\end{array}$ \\
\hline \multirow[t]{3}{*}{$\begin{array}{l}\text { Quality of } \\
\text { life }\end{array}$} & $\begin{array}{l}\text { Luo et al, } 2020^{15} \\
\text { Lee et al, } 2010^{16} \\
\text { Yan et al, 2014 } \\
\text { Zhang et al, } 2012^{19} \\
\text { Cramer et al, 2012 } \\
\text { Ford et al, } 2020^{23}\end{array}$ & $\begin{array}{l}* \\
\# \\
\# \\
* \\
* \\
*\end{array}$ & Pain & $\begin{array}{l}\text { Luo et al, } 2020^{15} \\
\text { Pan et al, } 2015^{17} \\
\text { Espíndula et al,2017 } \\
\text { Carral et al, } 2018^{32} \\
\text { Wayne et al, } 2017^{34} \\
\text { Pan et al, } 2016^{37}\end{array}$ & $\begin{array}{l}* \\
\# \\
* \\
* \\
\# \\
\# \\
\#\end{array}$ & BMI & $\begin{array}{l}\text { Pan et al, 2015 } \\
\text { Yan et al, 2014 } \\
\text { Liu et al, } 2020^{30} \\
\text { Zeng et al, 2014 } \\
\text { Pan et al, 2016 } \\
\text { Yan et al, 2013 }\end{array}$ & $\begin{array}{l}\# \\
\# \\
\# \\
\# \\
* \\
\#\end{array}$ \\
\hline & 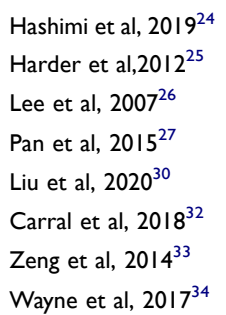 & $\begin{array}{l}\# \\
* \\
\# \\
* \\
* \\
* \\
* \\
*\end{array}$ & Anxiety & 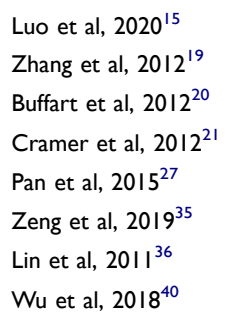 & $\begin{array}{l}* \\
\# \\
* \\
* \\
* \\
\# \\
* \\
* \\
*\end{array}$ & $\begin{array}{l}\text { Sleep } \\
\text { outcome }\end{array}$ & 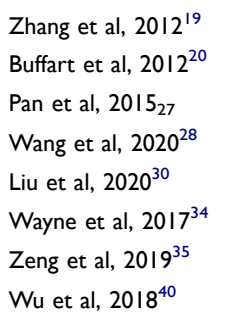 & $\begin{array}{l}\# \\
\# \\
* \\
* \\
\# \\
* \\
* \\
\#\end{array}$ \\
\hline & $\begin{array}{l}\text { Zeng et al, } 2019^{35} \\
\text { Pan et al, } 2016^{37} \\
\text { Yan et al, } 2013^{38} \\
\text { Wu et al,2018 }\end{array}$ & $\begin{array}{l}\# \\
\# \\
\# \\
*\end{array}$ & \multirow[t]{2}{*}{ Depression } & $\begin{array}{l}\text { Zhang et al, } 2012^{19} \\
\text { Buffart et al, } 2012^{20} \\
\text { Cramer et al, 2012 } \\
\text { Pan et al, 2015 }\end{array}$ & $\begin{array}{l}\# \\
* \\
* \\
*\end{array}$ & \multirow[t]{2}{*}{ Fatigue } & $\begin{array}{l}\text { Buffart et al, } 2012^{20} \\
\text { Dong et al, } 2019^{22} \\
\text { Pan et al, } 2015^{27} \\
\text { Sadja et al, } 2013^{29}\end{array}$ & $\begin{array}{l}\# \\
* \\
\# \\
*\end{array}$ \\
\hline Distress & $\begin{array}{l}\text { Zhang et al, } 2012^{19} \\
\text { Buffart et al, } 2012^{20} \\
\text { Lin et al, } 2011^{36}\end{array}$ & $\begin{array}{l}\# \\
* \\
*\end{array}$ & & $\begin{array}{l}\text { Liu et al, } 2020^{30} \\
\text { Zeng et al, } 2014^{33} \\
\text { Wayne et al, } 2017^{34} \\
\text { Lin et al, } 2011^{36} \\
\text { Wu et al, } 2018^{40}\end{array}$ & $\begin{array}{l}\# \\
\# \\
* \\
* \\
*\end{array}$ & & $\begin{array}{l}\text { Liu et al, } 2020^{30} \\
\text { Espíndula et al, } 2017^{31} \\
\text { Zeng et al, } 2019^{35} \\
\text { Zhang et al, } 2015^{39}\end{array}$ & $\begin{array}{l}\# \\
* \\
* \\
*\end{array}$ \\
\hline
\end{tabular}

Note: *, Alternative exercise traditions are useful; \#, Alternative exercise traditions are useless.

had certain defects, and 3 reports $(11.5 \%)$ had relatively serious information defects. Most of the researchers did not write a plan and performed registration before their systematic reviews, and the complete report rate of plans and registrations was only $11.5 \%$. In the results, only three articles (11.5\%) discussed the results of other analyses (such as sensitivity analysis or subgroups). In terms of the methodology, only five articles (19.2\%) described the bias in the evaluation of a single study (such as the suitability for random allocation sequence, blinding, and proportion of patients lost to follow-up). In conducting an SR/MA, the PRISMA statement should be used as basis in preparing a normative report to improve the overall report quality.

Therefore, report quality needs to be further improved. For example, the randomization and allocation concealment schemes included in the original study were not perfect, and the loss to follow-up and adverse events were not fully reported. Specific information, such as the individuals who conducted the search and those who implemented it and the details about resolving disagreements, was lacking.

\section{The Effectiveness of Alternative Exercise Traditions in Cancer Treatment Should be Discussed Further}

QOL is a multidimensional assessment with physical significance and is a commonly used indicator to measure the effect of cancer treatment. ${ }^{42}$ The $18 \mathrm{SR} / \mathrm{MAs}$ included in this study analyzed the intervention effects of alternative exercise traditions on QOL. Among the 18 articles, 11 reports showed that alternative exercise traditions can improve the QOL of cancer patients. Tai Chi, Qigong, yoga, and others that serve as traditional exercise activities can increase a person's strength, improve one's physical flexibility, and promote relaxation and well-being. ${ }^{24,42}$ Pain is one of the most common symptoms of cancer, and it may be due to tumors, surgery, chemotherapy, radiation therapy, targeted therapy, supportive care, and diagnostic procedures. ${ }^{43}$ The evaluation of control-related and nonsurgical pain is essential for cancer patients. From the 26 articles, 6 reports analyzed the intervention effects of alternative exercise traditions on pain, and 3 of them showed that alternative exercise traditions may not necessarily improve the pain of cancer patients. Pain has 
Table 3 Quality Assessment of the Included Reviews Using the AMSTAR Tool $(n=26)$

\begin{tabular}{|c|c|c|c|c|c|c|c|c|c|c|c|c|c|c|}
\hline \multirow[t]{2}{*}{ Authors, Year } & \multicolumn{11}{|c|}{ AMSTAR Items } & \multicolumn{3}{|c|}{ Total } \\
\hline & QI & Q2 & Q3 & Q4 & Q5 & Q6 & Q7 & Q8 & Q9 & Q10 & QII & $\mathbf{Y}$ & $\mathbf{N}$ & C \\
\hline Luo et al,2020 & Y & Y & Y & $\mathrm{N}$ & Y & Y & Y & Y & Y & Y & $N$ & 9 & 2 & 0 \\
\hline Lee et al, $2010^{16}$ & $\mathrm{~N}$ & Y & Y & $\mathrm{N}$ & Y & Y & Y & Y & $Y$ & $\mathrm{~N}$ & $N$ & 7 & 4 & 0 \\
\hline Pan et al, $2015^{17}$ & $\mathrm{~N}$ & $Y$ & $\mathrm{~N}$ & $\mathrm{~N}$ & Y & Y & Y & Y & Y & $\mathrm{N}$ & $\mathrm{N}$ & 6 & 5 & 0 \\
\hline Yan et al, $2014^{18}$ & $\mathrm{~N}$ & $\mathrm{~N}$ & $N$ & $\mathrm{~N}$ & Y & Y & Y & Y & Y & $Y$ & $\mathrm{~N}$ & 6 & 5 & 0 \\
\hline Zhang et al, $2012^{19}$ & $\mathrm{~N}$ & $Y$ & $Y$ & $Y$ & Y & Y & Y & Y & Y & Y & $Y$ & 10 & I & 0 \\
\hline Buffart et al,2012 20 & $\mathrm{~N}$ & $\mathrm{~N}$ & Y & $\mathrm{N}$ & Y & Y & Y & Y & Y & Y & Y & 8 & 3 & 0 \\
\hline Cramer et al,2012 & $\mathrm{N}$ & $Y$ & $\mathrm{~N}$ & $N$ & Y & Y & Y & Y & Y & Y & Y & 8 & 3 & 0 \\
\hline Dong et al, $2019^{22}$ & $N$ & Y & $Y$ & $\mathrm{~N}$ & Y & Y & Y & Y & Y & Y & Y & 9 & 2 & 0 \\
\hline Ford et al, $2020^{23}$ & $\mathrm{~N}$ & Y & $N$ & $Y$ & Y & Y & Y & Y & Y & Y & Y & 9 & 2 & 0 \\
\hline Hashimi et al,2019 $9^{24}$ & $\mathrm{~N}$ & Y & $Y$ & $\mathrm{~N}$ & Y & Y & Y & Y & Y & $\mathrm{N}$ & Y & 8 & 3 & 0 \\
\hline Harder et al, $2012^{25}$ & $\mathrm{~N}$ & $Y$ & Y & $\mathrm{N}$ & $Y$ & Y & Y & Y & Y & $\mathrm{N}$ & Y & 8 & 3 & 0 \\
\hline Lee et al, $2007^{26}$ & $\mathrm{~N}$ & $\mathrm{~N}$ & Y & $\mathrm{N}$ & $\mathrm{N}$ & Y & Y & Y & Y & $\mathrm{N}$ & $\mathrm{N}$ & 5 & 6 & 0 \\
\hline Pan et al, $2015^{27}$ & $\mathrm{~N}$ & $Y$ & $\mathrm{~N}$ & $\mathrm{~N}$ & $Y$ & Y & Y & Y & Y & $\mathrm{N}$ & $\mathrm{N}$ & 6 & 5 & 0 \\
\hline Wang et al, $2020^{28}$ & $\mathrm{~N}$ & Y & $\mathrm{N}$ & $\mathrm{N}$ & Y & Y & Y & Y & Y & $Y$ & $Y$ & 8 & 3 & 0 \\
\hline Sadja et al, $2013^{29}$ & $\mathrm{~N}$ & Y & $\mathrm{N}$ & $\mathrm{N}$ & Y & Y & Y & Y & $\mathrm{Y}$ & Y & $\mathrm{N}$ & 7 & 4 & 0 \\
\hline Liu et al, $2020^{30}$ & $\mathrm{~N}$ & Y & $Y$ & $\mathrm{~N}$ & Y & Y & Y & Y & $\mathrm{Y}$ & Y & $N$ & 8 & 3 & 0 \\
\hline Espíndula et al,201731 & $Y$ & Y & $N$ & $\mathrm{~N}$ & Y & Y & Y & Y & Y & Y & $\mathrm{N}$ & 8 & 3 & 0 \\
\hline Carral et al, $2018^{32}$ & $Y$ & Y & $N$ & $\mathrm{~N}$ & Y & Y & Y & Y & Y & Y & $Y$ & 9 & 2 & 0 \\
\hline Zeng et al, $2014^{33}$ & $\mathrm{~N}$ & Y & $N$ & $\mathrm{~N}$ & Y & Y & Y & Y & $Y$ & Y & Y & 8 & 3 & 0 \\
\hline Wayne et al, $2017^{34}$ & $\mathrm{~N}$ & Y & $\mathrm{N}$ & $\mathrm{N}$ & Y & Y & Y & Y & $Y$ & Y & Y & 8 & 3 & 0 \\
\hline Zeng et al, 201935 & $\mathrm{~N}$ & C & $\mathrm{N}$ & C & $\mathrm{N}$ & Y & Y & Y & Y & Y & Y & 6 & 3 & 2 \\
\hline Liu et al, $2011^{36}$ & $N$ & C & $\mathrm{N}$ & $\mathrm{N}$ & $Y$ & Y & $Y$ & Y & Y & $\mathrm{N}$ & $\mathrm{N}$ & 5 & 5 & 1 \\
\hline Pan et al, $2016^{37}$ & $\mathrm{~N}$ & $Y$ & $Y$ & $\mathrm{~N}$ & $\mathrm{~N}$ & Y & $\mathrm{N}$ & Y & Y & $Y$ & $\mathrm{~N}$ & 6 & 5 & 0 \\
\hline Yan et al, $2013^{38}$ & $\mathrm{~N}$ & Y & $Y$ & $\mathrm{~N}$ & $Y$ & Y & $Y$ & Y & $Y$ & $Y$ & $\mathrm{~N}$ & 8 & 3 & 0 \\
\hline Zhang et al, $2015^{39}$ & $\mathrm{~N}$ & Y & $N$ & $Y$ & Y & Y & Y & Y & Y & Y & $\mathrm{N}$ & 8 & 3 & 0 \\
\hline Wu et al, $2018^{40}$ & $\mathrm{~N}$ & $Y$ & $\mathrm{~N}$ & $\mathrm{~N}$ & $Y$ & $Y$ & $Y$ & Y & $Y$ & $\mathrm{~N}$ & $\mathrm{~N}$ & 6 & 5 & 0 \\
\hline $\begin{array}{l}\text { Percentage of systematic reviews meeting } \\
\text { each criteria }\end{array}$ & 11.5 & 80.8 & 42.3 & 11.5 & 88.5 & 100.0 & 96.2 & 100.0 & 100.0 & 69.2 & 46.2 & & & \\
\hline
\end{tabular}

Notes: AMSTAR items: Q1, a-priori design; Q2, duplicate study selection and data extraction; Q3, search comprehensiveness; Q4, status of publication (eg, non-English articles or dissertations); Q5, listing included and excluded studies; Q6, characteristics of the primary studies provided; Q7, scientific quality of the primary studies assessed and documented; Q8, scientific quality of the included data used appropriately in drawing conclusions; Q9, appropriateness of methods used to combine the findings of the primary studies; Q10, assessment of publication bias; and QII, acknowledgement of conflicts of interest and potential sources of support in both the systematic review and the primary studies. Y, yes; N, no; C, cannot answer. We assigned I point to each "yes" item. The sub-column "Y", the AMSTAR score, represents the quality of each included study which is the most important column in the table. The last row in the table indicates the percentage of included studies which met each item in AMSTAR scale. Bases on this, the methodological issues of included studies were identified.

a psychological impact as it may cause a patient to develop anxiety, neuroticism, and anger. ${ }^{44}$ A total of eight reports analyzed the intervention effects of alternative exercise traditions on anxiety, and six of them showed that alternative exercise traditions can improve anxiety in cancer patients. A total of nine reports analyzed the intervention effects of alternative exercise traditions on depression, and six of them showed that alternative exercise traditions can improve depression in cancer patients. A total of three reports analyzed the intervention effects of alternative exercise traditions on distress, and two of them showed that alternative exercise traditions can improve distress in cancer patients. In summary, alternative forms of exercise can be used as a supplementary therapy to help cancer patients with their social and psychological suffering. Tai Chi, Qigong, yoga, and others encourage patients to pay attention to their breathing, calm their mind, and concentrate. $^{45,46}$ For anxiety or depression that affects sleep, psychological counseling should be combined with physical and mental exercise. A total of eight reports analyzed the intervention effects of alternative exercise traditions on sleep outcomes, and four of them showed that alternative exercise traditions may not necessarily improve the sleep outcomes of cancer patients. Sleep disorders are known to be related to inflammation. ${ }^{47}$ Studies have shown that exercise is an effective intervention to 


\begin{tabular}{|c|c|c|c|c|c|c|c|c|c|c|c|c|c|}
\hline 宽 & - & $\stackrel{\text { นุ }}{0}$ & -- & $\circ$ & $-\ldots$ & - 旾 & - - 奋 & $\circ$ & - - 年 & - & - 婠 & 。 & -- \\
\hline 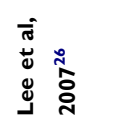 & - & $\stackrel{n}{0}$ & $\stackrel{n}{o}-$ & 。 & - 㔯。资。 & - 吕 & --0 & $\circ$ & $\stackrel{n}{o}-\stackrel{n}{0}$ & $\circ$ & 00 & $\circ$ & $\stackrel{n}{\circ}-$ \\
\hline 离 & - & นn & 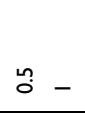 & $\circ$ & 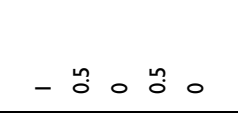 & $-\stackrel{n}{0}$ & --0 & $\circ$ & - - ํㅗㅇ & นn & นุำ ถุ & $\circ$ & - 号 - \\
\hline 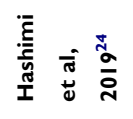 & - & นุ & $\stackrel{n}{0}-$ & ○ & 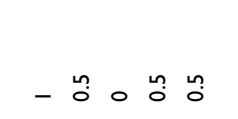 & - - n̊ & - - מํํ & 。 & $\stackrel{n}{o}-\stackrel{n}{o}$ & - & - ํํㅇ & 。 & -- \\
\hline 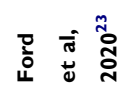 & - & นุ & $\stackrel{n}{0}-$ & ○ & - 荢 - 号 - & - - n̊ & -- & 。 & --- & - & - 望 & 。 & -- \\
\hline 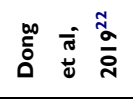 & - & นn & -- & $\circ$ & $-\ldots$ - n & - กั & --- & $\circ$ & $\stackrel{n}{\circ}--$ & - & -- & $\circ$ & $-\stackrel{n}{0}-$ \\
\hline 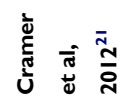 & - & นn & -- & $\circ$ & $-\ldots$ & - n & - - מํํ & $\circ$ & --- & - & -- & 。 & - $\stackrel{\text { on }-}{-}$ \\
\hline 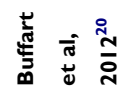 & - & นn & $\stackrel{n}{o}-$ & 0 & 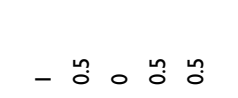 & -0 & - - นุำ & 0 & --0 & นn & นุํ & 。 & $\stackrel{n}{o}-$ \\
\hline 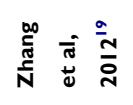 & - & นn & $\stackrel{n}{0}-$ & ○ & - 茖㔯 - - & - n̊ & - - ถุ & 。 & - - ถุ & - & - 望 & 。 & -- \\
\hline 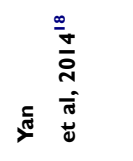 & - & นุ & $\stackrel{n}{o}-$ & $\circ$ & - นุㅇำ & - 足 & -- & $\circ$ & $\stackrel{n}{o}-\stackrel{n}{o}$ & - & $-\stackrel{n}{0}$ & $\circ$ & $-\stackrel{n}{\circ}-$ \\
\hline 自 & - & n̊ & -- & $\circ$ & $--0--$ & - ? & - - 㔯 & n̊ & - - 号 & - & $-\stackrel{n}{0}$ & $\circ$ & -- \\
\hline 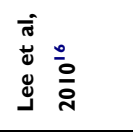 & - & นn & $\stackrel{n}{\circ}-$ & 。 & 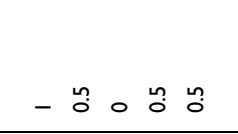 & - $\stackrel{\text { on }}{0}$ & - - นุ & 。 & - - ڤn & - & - นٌ & 。 & - $\stackrel{\text { o }}{0}$ \\
\hline 马 & - & - & $\stackrel{n}{\circ}-$ & - & $--0-\stackrel{n}{0}$ & $-\stackrel{n}{0}$ & --- & - & --- & - & 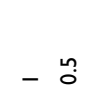 & - & -- \\
\hline 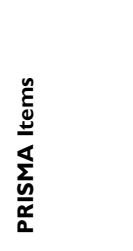 & 兽 & 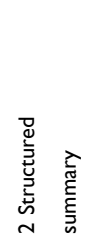 & 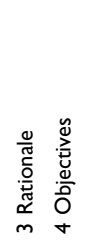 & 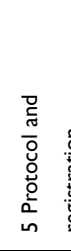 & 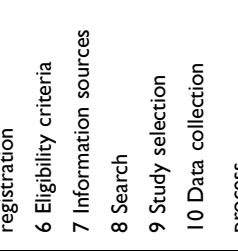 & 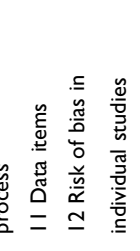 & 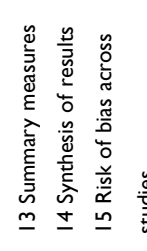 & 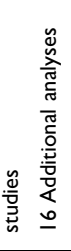 & 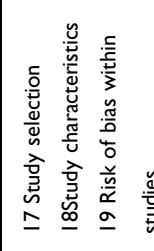 & 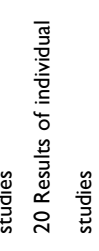 & 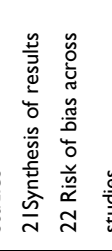 & 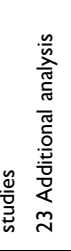 & 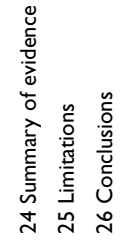 \\
\hline$\stackrel{\underline{0}}{\circ}$ & $\stackrel{0}{\rightleftarrows}$ & $\begin{array}{l}\text { 䔍 } \\
\text { 产 }\end{array}$ & 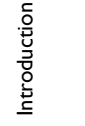 & 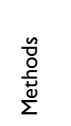 & & & & & $\begin{array}{l}\stackrel{\underline{y}}{\bar{z}} \\
\propto \\
\propto\end{array}$ & & & & 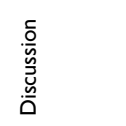 \\
\hline
\end{tabular}




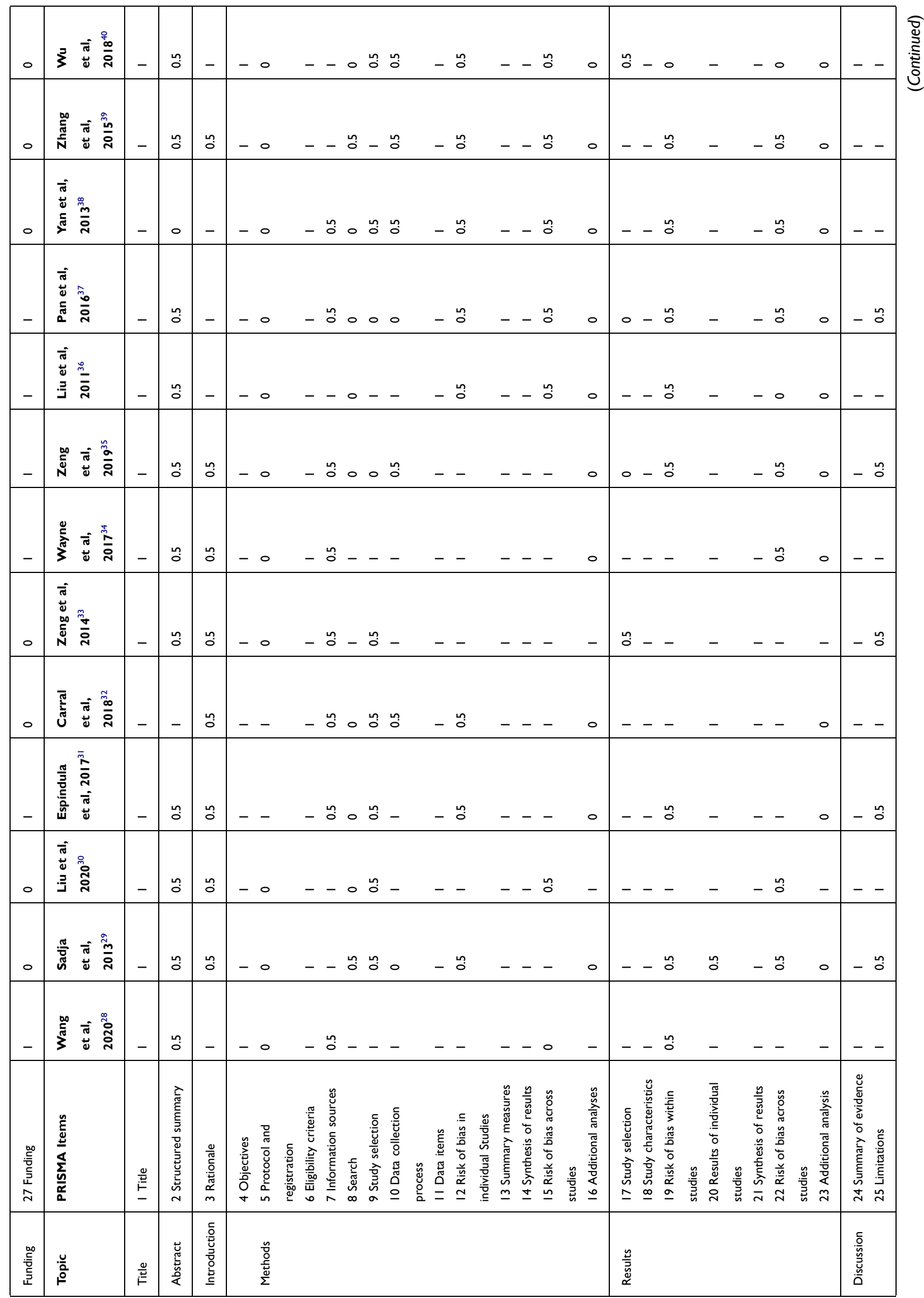




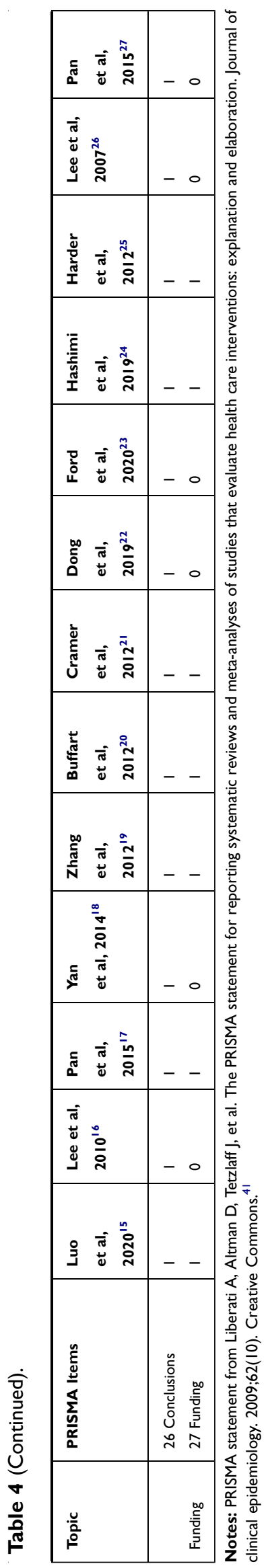

control inflammation, ${ }^{48}$ but the mechanism of how exercise affects sleep is still unclear. At the same time, due to insufficient intensity, exercise may not trigger changes that promote better sleep (such as body changes, melatonin release, and reduction of inflammation). A total of six reports included in this study analyzed the intervention effects of alternative exercise traditions on BMI. Of these reports, five showed that alternative exercise traditions cannot reduce the BMI of cancer patients. This result may be related to differences in race, region, and age. ${ }^{49-51}$ A proper extension of exercise time may be effective in reducing the BMI of cancer patients. ${ }^{52}$ At the same time, BMI reflects the overall body weight and does not distinguish between adipose tissue and nonfat tissue. ${ }^{53,54}$ In the future, other physical measurement indicators for evaluating obesity are expected. A total of eight reports analyzed the intervention effects of alternative exercise traditions on fatigue, and five of them showed that alternative exercise traditions can improve fatigue in cancer patients. Alternative exercise traditions integrate exercise (body posture), meditation (concentration), and breathing to achieve a state of mental calm and relaxation to reduce fatigue. ${ }^{55-57}$

In summary, alternative exercise traditions have the potential to improve the negative psychological conditions of cancer patients and improve their QOL. However, whether or not they are more advantageous than conventional rehabilitation training still needs to be supported by a large sample size and strict standardized trials. At the same time, Tai Chi, yoga, and Qigong cannot be used as intervention methods in general without distinguishing the characteristics of different schools and movements. Future research should incorporate a large number of samples (in the baseline and follow-up phases), long-term follow-up evaluations (eg, 6 months or more), and clearly defined targeted measurement indicators into the design.

\section{Alternative Exercise Traditions in the Context of the COVID-19 Pandemic may be an Effective Intervention That is Easy to Promote}

COVID-19 is the most widespread global pandemic to affect humans in the last 100 years. ${ }^{58}$ In the early stage of the epidemic, academician Zhong Nanshan spoke highly of Tai Chi for strengthening one's body and fitness. ${ }^{59}$ In particular, Tai Chi has been regarded as capable of improving cardiopulmonary function, thereby 
attracting people's attention. ${ }^{60}$ The National Health Commission of the People's Republic of China issued the "Rehabilitation Plan for Discharged COVID-19 Patients (Trial)" on March 4, 2020.61 The section on "Rehabilitation treatment methods" pointed out that a series of aerobic prescriptions have been formulated for patients with combined underlying diseases and residual dysfunction. These prescriptions include Tai Chi and Baduan Jin. According to the plan, patients are encouraged to begin with low-intensity exercise and progress gradually, engaging in exercise for 20-30 min each time and 3-5 times a week. The appropriate exercise intensity is one that does not cause fatigue the next day.

COVID-19 is not only a public health incident but also a psychological crisis. $^{62}$ The UK Household Longitudinal Study (UKHLS) revealed that 1 month after the implementation of the British football ban, the prevalence of people with clinically significant mental pain rose from $18.9 \%$ in $2018-2019$ to $27.3 \%$ in April 2020.6 ${ }^{63}$ In cancer patients, reduced parasympathetic tone may also be related to a long disease course and short survival. ${ }^{64,65}$ Yoga, Tai Chi, Qigong, and others can change the structure of the brain, regulate physiological activities, help alleviate psychological pressure, improve autonomic nervous system balance, ${ }^{66,67}$ and regulate sympathy by improving central activity under the stress-parasympathetic nerve output. Practicing Tai Chi and Qigong may reduce the sympathetic nerve tension, increase the parasympathetic nerve tension, and improve the peripheral autonomic nerve activity. ${ }^{68,69}$ In addition, the training content of alternative exercise traditions is simple and requires minimal equipment and venues. In the context of the COVID-19 pandemic, these forms of exercise are safer and more natural than other sports activities. Moreover, they may be easy to promote and can alleviate the subhealth of the public.

In summary, this study re-evaluated the published SR/ MAs of alternative exercise traditions for cancer treatment and provided an important reference for the application of alternative forms of exercise in the field of cancer treatment. However, as this study only included Chinese and English literature, the research conclusions obtained may have certain limitations. Moreover, the methodological quality and standardized treatment reporting of the SR/ MAs included in this study need to be improved. Researchers are recommended to strictly follow the AMSTAR scale and PRISMA statement when writing SR/MAs so as to provide high-quality research and provide a scientific and reliable reference for clinical diagnosis and treatment decisions. The topic of alternative exercise tradition for cancer intervention is still a hotspot in international research. Researchers can pay close attention to the improvement of the preliminary design plan, publication status, literature search, conflict of interest, etc.

\section{Conclusions}

In conclusion, the methodological quality of the systematic evaluations of the intervention effects of alternative exercise traditions on cancer patients is not highly satisfactory. Therefore, we suggest that focus should be directed to the improvement of the preliminary design scheme, publication status, literature retrieval, conflict of interest, and other aspects. Under the current situation of strict prevention and control of the epidemic and the promotion of selfisolation at home, alternative exercise traditions should be encouraged and promoted as early as possible. Professionals can develop appropriate exercise alternatives to ensure the expected effect of exercise while ensuring the safety of patients. At the same time, the application of alternative exercise traditions to the prevention of COVID19, its auxiliary treatment, and the rehabilitation of affected patients should be strengthened so as to continuously optimize the program.

\section{Acknowledgments}

This study was supported by Zhangjiagang City Science and Technology Support Program (Social Development) [ZKS1924]; Zhangjiagang City Health Youth Science and Technology Program [ZJGQNKJ202009]; Hospital-level Program of Zhangjiagang Hospital Affiliated to Nanjing University of Chinese Medicine[zzyq1905].

\section{Disclosure}

The authors have declared that they have no competing interests.

\section{References}

1. Desales E, Khusro A, Cipriano-Salazar M, Barbabosa-Pliego A, RivasCaceres RR. Scorpion venoms and associated toxins as anticancer agents: update on their application and mechanism of action. $J$ Appl Toxicol. 2020;40(10):1-15. doi:10.1002/jat.3976.

2. Bray F, Ferlay J, Soerjomataram I, Siegel RL, Torre LA, Jemal A. Global cancer statistics 2018: GLOBOCAN estimates of incidence and mortality worldwide for 36 cancers in 185 countries. CA Cancer J Clin. 2018;68(6):394-424. doi:10.3322/caac.21492.

3. Varghese C, Carlos MC, Shin HR. Cancer burden and control in the western pacific region: challenges and opportunities. Ann Global Health. 2014;80(5):358-369. doi:10.1016/j.aogh.2014.09.015. 
4. Siegel RL, Miller KD, Jemal A. Cancer statistics, 2016. CA Cancer J Clin. 2016;66(1):7-30. doi:10.3322/caac.21332.

5. Global Burden of Disease Cancer Collaboration. Global, regional, and national cancer incidence, mortality, years of life lost, years lived with disability, and disability-adjusted life-years for 32 Cancer Groups, 1990 to 2015 a systematic analysis for the global burden of disease study. JAMA Oncol. 2017;3(4):524-548. doi:10.1001/ jamaoncol.2016.5688.

6. Sharma P, McClees SF, Afaq F. Pomegranate for prevention and treatment of cancer: an update. Molecules. 2017;22(1):177. doi: $10.3390 /$ molecules 22010177 .

7. World Health Organization. [homepage on the Internet]. Available from: https://www.who.int/. Accessed Novermber 10, 2020. (Published in English).

8. Liang W, Guan W, Chen R, et al. Cancer patients in SARS-CoV-2 infection: a nationwide analysis in China. Lancet Oncol. 2020;21 (3):335-337. doi:10.1016/S1470-2045(20)30096-6.

9. Newton RU, Hart N, Clay T. Keeping cancer patients exercising in the age of COVID-19. J Oncol Pract. 2020;16(10):1-10. doi:10. 1200/OP.20.00210.

10. Hongyu. Hospital in Wuhan uses TCM to treat novel coronavirus patients. People's Daily Online. 2020. Available from: http://en.people.cn/n3/2020/ 0228/c98649-9663201.html. Accessed Novermber 10, 2020.

11. Yao D, Zhang YJ, Wang JQ. [Thought on Traditional Chinese Medicine Traditional Methods from Modern Research-Baduanjin in the Prevention and Treatment of COVID-19]. Chin Med Modern Distance Educ China. 2020;18(5):39-41. (Published in Chinese).

12. Han JL, Gandhi S, Bockoven CG, Narayan VM, Dahm P. The landscape of systematic reviews in urology (1998 to 2015): an assessment of methodological quality. BJU Int. 2017;119(4):638-649. doi:10.1111/bju.13653.

13. Kitsiou S, Paré G, Jaana M, Gerber B. Effectiveness of mHealth interventions for patients with diabetes: an overview of systematic reviews. PLoS One. 2017;12(3):e0173160. doi:10.1371/journal. pone. 0173160 .

14. Zhou W, Ge L, Xu J, et al. [Randomized controlled trial quality evaluation on the systematic reviews/meta-analyses related to interventions published in the Chinese Journal of Evidence-Based Medicine]. Chin J Evid-Based Med. 2013;13(4):482-488. (Published in Chinese).

15. Luo XC, Liu J, Fu J, et al. Effect of Tai Chi Chuan in breast cancer patients: a systematic review and meta-analysis. SSRN Elect J. 2020;10:607. doi:10.2139/ssrn.3381147.

16. Lee MS, Choi TY, Ernst E. Tai chi for breast cancer patients: a systematic review. Breast Cancer Res Treat. 2010;120 (2):309-316. doi:10.1007/s10549-010-0741-2.

17. Pan Y, Yang K, Shi X, Liang H, Zhang F, Lv Q. Tai chi chuan exercise for patients with breast cancer: a systematic review and meta-analysis. Evid Based Complement Alternat Med. 2015;2015:535237. doi:10.1155/2015/535237.

18. Yan JH, Pan L, Zhang XM, Sun CX, Cui GH. Lack of efficacy of Tai Chi in improving quality of life in breast cancer survivors: a systematic review and meta-analysis. Asian Pac J Cancer Prev. 2014;15(8):3715-3720. doi:10.7314/apjcp.2014.15.8.3715.

19. Zhang J, Yang KH, Tian JH, Wang CM. Effects of yoga on psychologic function and quality of life in women with breast cancer: a meta-analysis of randomized controlled trials. J Altern Complement Med. 2012;18(11):994-1002. doi:10.1089/acm.2011.0514.

20. Buffart LM, Uffelen J, Riphagen II, et al. Physical and psychosocial benefits of yoga in cancer patients and survivors, a systematic review and meta-analysis of randomized controlled trials. BMC Cancer. 2012;12:559. doi:10.1186/1471-2407-12-559.

21. Cramer H, Lange S, Klose P, Paul A, Dobos G. Yoga for breast cancer patients and survivors: a systematic review and meta-analysis. BMC Cancer. 2012;12:412. doi:10.1186/1471-2407-12-412
22. Dong B, Xie C, Jing X, Lin L, Tian L. Yoga has a solid effect on cancer-related fatigue in patients with breast cancer: a meta-analysis. Breast Cancer Res Treat. 2019;177(1):5-16. doi:10.1007/s10549-01905278-w

23. Ford CG, Vowles KE, Smith BW, Kinney AY. Mindfulness and meditative movement interventions for men living with cancer: a meta-analysis. Ann Behav Med. 2020;54(5):360-373. doi:10.1093/ $\mathrm{abm} / \mathrm{kaz} 053$

24. El-Hashimi D, Gorey KM. Yoga-specific enhancement of quality of life among women with breast cancer: systematic review and exploratory meta-analysis of randomized controlled trials. J Evid Based Integr Med. 2019;24:1-9. doi:10.1177/2515690x19828325

25. Harder H, Parlour L, Jenkins V. Randomised controlled trials of yoga interventions for women with breast cancer: a systematic literature review. Support Care Cancer. 2012;20(12):3055-3064. doi:10.1007/ s00520-012-1611-8

26. Lee MS, Pittler MH, Ernst E. Is Tai Chi an effective adjunct in cancer care? A systematic review of controlled clinical trials. Support Care Cancer. 2007;15(6):597-601. doi:10.1007/s00520-007-0221-3

27. Pan Y, Yang K, Wang Y, Zhang L, Liang H. Could yoga practice improve treatment-related side effects and quality of life for women with breast cancer? A systematic review and meta-analysis. Asia Pac J Clin Oncol. 2017;13(2):e79-e95. doi:10.1111/ajco.12329

28. Wang WL, Chen KH, Pan YC, Yang SN, Chan YY. The effect of yoga on sleep quality and insomnia in women with sleep problems: a systematic review and meta-analysis. BMC Psychiatry. 2020;20 (1):195. doi:10.1186/s12888-020-02566-4

29. Sadja J, Mills PJ. Effects of yoga interventions on fatigue in cancer patients and survivors: a systematic review of randomized controlled trials. Explore (NY). 2013;9(4):232-243. doi:10.1016/j.explore.2013. 04.005

30. Liu L, Tan H, Yu S, Yin H, Baxter GD. The effectiveness of Tai Chi in breast cancer patients: a systematic review and meta-analysis. Complement Ther Clin Pract. 2020;38:101078. doi:10.1016/j. ctcp.2019.101078

31. Espíndula RC, Nadas GB, Rosa MID, Foster C, Araújo FC, Grande AJ. Pilates for breast cancer: a systematic review and meta-analysis. Rev Assoc Med Bras. 2017;63(11):1006-1012. doi:10.1590/1806-9282.63.11.1006

32. Pinto-Carral A, Molina AJ, de Pedro Á, Ayán C. Pilates for women with breast cancer: a systematic review and meta-analysis. Complement Ther Med. 2018;41:130-140. doi:10.1016/j.ctim.2018.09.011

33. Zeng Y, Luo T, Xie H, Huang M, Cheng AS. Health benefits of qigong or Tai Chi for cancer patients: a systematic review and meta-analyses. Complement Ther Med. 2014;22(1):173-186. doi:10.1016/j.ctim.2013.11.010

34. Wayne PM, Lee MS, Novakowski J, et al. Tai Chi and Qigong for cancer-related symptoms and quality of life: a systematic review and meta-analysis. J Cancer Surviv. 2018;12(2):256-267. doi:10.1007/ s11764-017-0665-5

35. Zeng Y, Xie X, Cheng ASK. Qigong or Tai Chi in cancer care: an updated systematic review and meta-analysis. Curr Oncol Rep. 2019;21(6):48. doi:10.1007/s11912-019-0786-2

36. Lin KY, Hu YT, Chang KJ, Lin HF, Tsauo JY. Effects of yoga on psychological health, quality of life, and physical health of patients with cancer: a meta-analysis. Evid Based Complement Alternat Med. 2011;2011:659876. doi:10.1155/2011/659876

37. Pan YQ, Shi XE, Yao XR, et al. [Systematic review on effect of Tai Chi Chuan rehabilitative therapy on side effects of postoperative patients with breast cancer]. J Lanzhou Univ. 2016;42(3):64-71. (Published in Chinese).

38. Yan LJ, Cao HJ, Hao YF. [Effect of Tai Chi on quality of life of patients with breast cancer: a systematic review of randomized controlled trials]. Chin J Rehabil Theory Pract. 2013;19(6):592-597. (Published in Chinese). 
39. Zhang Q, Piao L, Zhao DM, Wu X, Cong YF. [Effects of Yoga on cancer-related fatigue in breast cancer patients: meta analysis]. Chin J Mod Nurs. 2015;28:3380-3386. (Published in Chinese).

40. Wu Q, Yin YT, Chen LJ, Li YL, Qiao YJ. [Effects of yoga on negative emotions and quality of life in patients with breast cancer: a meta-analysis]. Chin J Med. 2018;53(5):559-564. (Published in Chinese).

41. PRISMA figure adapted from Liberati A, Altman D, Tetzlaff J, et al. The PRISMA statement for reporting systematic reviews and metaanalyses of studies that evaluate health care interventions: explanation and elaboration. Journal of clinical epidemiology. 2009;62(10). Creative Commons.

42. Victorson D, Cella D, Wagner L, Kramer L, Smith ML. Measuring quality of life in cancer survivors. Handbook of Cancer Survivorship. In: Feuerstein M, editor. Handbook of Cancer Survivorship. Boston, MA: Springer; 2007:79-110.

43. National Cancer Institute. Cancer Pain (PDQ)-Health Professional Version (2020). Available from: https://www.cancer.gov/about-cancer/treatment/ side-effects/pain/pain-hp-pdq. Accessed Novermber 10, 2020.

44. Butler LD, Koopman C, Neri E, et al. Effects of supportive-expressive group therapy on pain in women with metastatic breast cancer. Health Psychol. 2009;28(5):579-587. doi:10.1037/a0016124

45. Harrison AM, Scott W, Johns LC, Morris EMJ, McCracken L. Are we speaking the same language? Finding theoretical coherence and precision in "mindfulness-based mechanisms" in chronic pain. Pain Med. 2017;18(11):2138-2151. doi:10.1093/pm/pnw310

46. Chiesa A, Malinowski P. Mindfulness-based approaches: are they all the same? J Clin Psychol. 2011;67(4):404-424. doi:10.1002/ jclp. 20776

47. Milrad SF, Hall DL, Jutagir DR, et al. Poor sleep quality is associated with greater circulating pro-inflammatory cytokines and severity and frequency of chronic fatigue syndrome/myalgic encephalomyelitis (CFS/ME) symptoms in women. J Neuroimmunol. 2017;303:43-50. doi:10.1016/j.jneuroim.2016.12.008

48. Meneses-Echávez JF, Correa-Bautista JE, González-Jiménez E, et al. The effect of exercise training on mediators of inflammation in breast cancer survivors: a systematic review with meta-analysis. Cancer Epidemiol Biomarkers Prev. 2016;25(7):1009-1017. doi:10.1158/ 1055-9965.Epi-15-1061

49. Rosner B, Eliassen AH, Toriola AT, et al. Weight and weight changes in early adulthood and later breast cancer risk. Int J Cancer. 2017;140 (9):2003-2014. doi:10.1002/ijc.30627

50. John EM, Sangaramoorthy M, Phipps AI, Koo J, Horn-Ross PL. Adult body size, hormone receptor status, and premenopausal breast cancer risk in a multiethnic population: the San Francisco Bay area breast cancer study. Am J Epidemiol. 2010;173(2):201-216. doi:10.1093/aje/kwq345

51. Amadou A, Ferrari P, Muwonge R, et al. Overweight, obesity and risk of premenopausal breast cancer according to ethnicity: a systematic review and dose-response meta-analysis. Obes Rev. 2013;14(8):665-678. doi:10.1111/obr.12028

52. He JH, Yao L, Chang Z, Liu GN. [Rehabilitation effect of systematic exercise in adjuvant chemotherapy for breast cancer patients]. Chin Rehabil. 2011;26(3):204-206. (Published in Chinese)

53. Yajnik CS, Yudkin JS. The Y-Y paradox. Lancet. 2004;363 (9403):163. doi:10.1016/S0140-6736(03)15269-5

54. Merry AHH, Schouten LJ, Goldbohm RA, Brandt P. Body mass index, height and risk of adenocarcinoma of the oesophagus and gastric cardia: a prospective cohort study. Gut. 2007;56(44):1503-1511. doi:10.1136/ gut.2006.116665
55. Greenlee H, DuPont-Reyes MJ, Balneaves LG, et al. Clinical practice guidelines on the evidence-based use of integrative therapies during and after breast cancer treatment. CA Cancer J Clin. 2017;67 (3):194-232. doi:10.3322/caac.21397

56. Jahnke R, Larkey L, Rogers C, Etnier J, Lin F. A comprehensive review of health benefits of qigong and Tai Chi. Am J Health Promot. 2010;24(6):e1-e25. doi:10.4278/ajhp.081013-LIT-248

57. Mazzarino M, Kerr D, Wajswelner H, Morris ME. Pilates method for women's health: systematic review of randomized controlled trials. Arch Phys Med Rehabil. 2015;96(12):2231-2242. doi:10.1016/j. apmr.2015.04.005

58. Ragab D, Eldin HS, Taeimah M, Khattab R, Salem R. The COVID-19 cytokine storm; what we know so far. Front Immunol. 2020;11:1446. doi:10.3389/fimmu.2020.01446

59. Xi'an wang. Academician Zhong Nanshan Talks about Traditional Chinese Medicine and Taijiquan. Sina News. 2020. Available from: http://k.sina.com.cn/article_2618630987_m9c15234b03300n2ok. html. Accessed Novermber 10, 2020.

60. Sun L, Zhuang L, Li X, Zheng J. Tai Chi can prevent cardiovascular disease and improve cardiopulmonary function of adults with obesity aged 50 years and older: a long-term follow-up study. Medicine. 2019;98(42):e17509. doi:10.1097/MD.0000000000017509

61. National Health Commission of the People's Republic of China. Notice of the General Office of the National Health Commission on Issuing the Rehabilitation Plan (Trial) for Discharged Patients with New Coronary Pneumonia National Health Office Medical Letter (2020) No. 189. 2020. Available from: http://www.nhc.gov.cn/yzygj/ s7653pd/202003/d4558d2cc35e44d5b9adba7c911e0b4c.shtml.

62. Zgueb Y, Bourgou S, Neffeti A, et al. Psychological crisis intervention response to the COVID 19 pandemic: a Tunisian centralised protocol. Psychiat Res. 2020;289:113042. doi:10.1016/j.psychres.2020.113042

63. Pierce M, Hope H, Ford T, et al. Mental health before and during the COVID-19 pandemic: a longitudinal probability sample survey of the UK population. Lancet Psychiat. 2020;7(10):883-892. doi:10.1016/ s2215-0366(20)30308-4

64. Giese-Davis J, Wilhelm FH, Tamagawa R, et al. Higher vagal activity as related to survival in patients with advanced breast cancer: an analysis of autonomic dysregulation. Psychosom Med. 2015;77 (4):346-355. doi:10.1097/psy.0000000000000167

65. Couck MD, Maréchal R, Moorthamers S, Van Laethem JL, Gidron Y. Vagal nerve activity predicts overall survival in metastatic pancreatic cancer, mediated by inflammation. Cancer Epidemiol. 2016;40:47-51. doi:10.1016/j.canep.2015.11.007

66. Bamber MD, Schneider JK. Mindfulness-based meditation to decrease stress and anxiety in college students: a narrative synthesis of the research. Educ Res Rev. 2016;18:1-32. doi:10.1016/j.edurev.2015.12.004

67. Travis F, Haaga DA, Hagelin J, et al. Effects of Transcendental Meditation practice on brain functioning and stress reactivity in college students. Int $J$ Psychophysiol. 2009;71(2):170-176. doi:10.1016/j.ijpsycho.2008.09.007

68. Ramos CC, França E, Nobre TL, et al. Qi Gong and heart rate variability: a systematic review. J Biosci Med. 2017;5(3):120-127. doi:10.4236/jbm.2017.53013

69. Cole AR, Wijarnpreecha K, Chattipakorn SC, Chattipakorn N. Effects of Tai Chi exercise on heart rate variability. Complement Ther Clin Pract. 2016;23:59-63. doi:10.1016/j.ctcp.2016.03.007 


\section{Publish your work in this journal}

Cancer Management and Research is an international, peer-reviewed open access journal focusing on cancer research and the optimal use of preventative and integrated treatment interventions to achieve improved outcomes, enhanced survival and quality of life for the cancer patient.
The manuscript management system is completely online and includes a very quick and fair peer-review system, which is all easy to use. Visit http://www.dovepress.com/testimonials.php to read real quotes from published authors.

Submit your manuscript here: https://www.dovepress.com/cancer-management-and-research-journal 